Supporting Information

\title{
Poultry Waste Valorization via Pyrolysis Technologies: Economic and Environmental Life Cycle Optimization for Sustainable Bioenergy Systems
}

\author{
Ning Zhao ${ }^{1}$, Johannes Lehmann ${ }^{2,3}$, Fengqi You ${ }^{1,3,4, *}$ \\ ${ }^{1}$ Systems Engineering, Cornell University, Ithaca, New York, 14853, USA \\ ${ }^{2}$ Soil and Crop Sciences, School of Integrated Plant Sciences, College of Agriculture and Life \\ Sciences, Cornell University, Ithaca, New York 14853, USA \\ ${ }^{3}$ Atkinson Center for a Sustainable Future, Cornell University, Ithaca, New York, 14853, USA \\ ${ }^{4}$ Robert Frederick Smith School of Chemical and Biomolecular Engineering, Cornell University, \\ Ithaca, New York, 14853, USA
}

Tuesday, March 3, 2020

Submitted to ACS Sustainable Chemistry \& Engineering

Number of Pages: 24

Number of Figures: 2

Number of Tables: 5

* Corresponding author. Phone: (607) 255-1162; Fax: (607) 255-9166; E-mail: fengqi.you@cornell.edu 


\section{Model Formulation}

Following the problem statement, a multi-objective MILFP model is proposed in this section, which addresses the LCO of waste biomass supply chain for the poultry sector considering pyrolysis technologies.

The two conflicting objectives introduced in the previous section are represented by Eq. (49) and Eq. (56) that stand for the unit annualized profit objective and the unit environmental impact objective, respectively. Constraints (1) - (3) are poultry waste feedstock supply system constraints. Constraints (4) - (23) are constraints for pyrolysis facilities. Constraints (24) - (38) are upgrading facility constraints. Product distribution system constraints are constraints (39) and (40). Corresponding economic and environmental constraints are constraints (41) - (48) and constraints (50) - (55), respectively.

All sets, indices, variables and parameters that are involved in the model are summarized and listed in the Nomenclature. All variables are denoted with lower-case symbols; all parameters and sets are denoted with upper case symbols. The LCO model of the poultry litter supply chain considering pyrolysis technologies is formulated in the following subsections.

\section{Constraints}

\section{Poultry waste feedstock supply system}

Constraint (1) shows the upper limit of poultry waste acquisition. The procured poultry waste amount at a specific poultry farm and a specific time period cannot exceed the available amount of this type of waste biomass. The set of poultry waste types indexed by $b$ is denoted as $B$. In addition, $I$ denotes the set of feedstock poultry farms, and $T$ represents the set of time periods.

$$
b m p_{b, i, t} \leq B A_{b, i, t}, \forall b \in B, i \in I, t \in T
$$

where $b m p_{b, i, t}$ is the amount of poultry waste type $b$ procured from poultry farm $i$ in time period $t$. Parameter $B A_{b, i, t}$ is the amount of poultry waste type $b$ available at poultry farm $i$ in time period $t$. It is worth noting that seasonality and geographical availability of different types of waste can be represented by different values of $B A_{b, i, t}$.

Constraint (2) represents the mass balance of the organic waste transportation process. The set of pyrolysis facilities indexed by $k$ is denoted as $K$, and $M$ is the set of transportation modes. 


$$
b m p_{b, i, t}=\sum_{k} \sum_{m} f i k_{b, i, k, m, t}, \forall b \in B, i \in I, t \in T
$$

where $f i k_{b, i, k, m, t}$ represents the dry weight of poultry waste type $b$ shipped from poultry farm $i$ to pyrolysis facilities $k$ using transportation mode $m$ in time period $t$. The amount of poultry litter acquired $\left(b m p_{b, i, t}\right)$ equals to the total amount shipped to pyrolysis facilities $\left(\sum_{k} \sum_{m} f i k_{b, i, k, m, t}\right)$.

Constraint (3) stands for transportation capacity constraints. $\rho_{b}$ is the mass of standard dry biomass of 1 dry ton of poultry waste type $b$, and $M C_{b}$ is the moisture content of poultry waste type $b . W C I K_{i, k, m, t}$ represents the feedstock transportation capacity from poultry farm $i$ to pyrolysis facility $k$ using transportation mode $m$ in time period $t$. The total shipping amount of organic waste (left-hand side) should not exceed the corresponding transportation capacity $\left(W C I K_{i, k, m, t}\right)$.

$$
\sum_{b} \frac{\rho_{b} f i k_{b, i, k, m, t}}{1-M C_{b}} \leq W C I K_{i, k, m, t}, \forall i \in I, k \in K, m \in M, t \in T
$$

\section{Pyrolysis facilities}

Constraints (4) and (5) represent the mass balance of poultry litter feedstock in pyrolysis facilities. $\varepsilon_{b, t}$ is the proportion of waste type $b$ deteriorated during storage in time period $t$. $s b k_{b, k, t}$ is the storage level of poultry litter $b$ at pyrolysis facility $k$ in time period $t$. The set of pyrolysis technologies (indexed by $q$ ) and intermediate upgrading technologies (indexed by $q^{\prime}$ ) is denoted by $Q$, and $w b k_{b, k, q, t}$ represents the amount of poultry waste type $b$ processed through technology $q$ in pyrolysis facility $k$ in time period $t$. As constraints (4) and (5) show, the poultry waste "input" in time period $t$ is the left-hand side, which equals to the summation of the amount of poultry litter shipped to a pyrolysis facility $\left(\sum_{i} \sum_{m} f i k_{b, i, k, m, t}\right)$, and the remaining feedstock storage amount ( $\left.\left(1-\epsilon_{b, t}\right) s b k_{b, k, t-1}\right)$. On the other hand, the poultry litter "output" in time period $t$ are shown on the right-hand side, which is the summation of the amount of litter processed through pyrolysis ( $\left.\sum_{q} w b k_{b, k, q, t}\right)$ and the storage amount of litter $\left(s b k_{b, k, t}\right)$.

$$
\begin{aligned}
& \sum_{i} \sum_{m} f i k_{b, i, k, m, t}+\left(1-\epsilon_{b, t}\right) s b k_{b, k, t-1}=\sum_{q} w b k_{b, k, q, t}+s b k_{b, k, t}, \forall b \in B, k \in K, t \geq 2 \\
& \sum_{i} \sum_{m} f i k_{b, i, k, m, t=1}+\left(1-\epsilon_{b, t=1}\right) s b k_{b, k, t=|T|}=\sum_{q} w b k_{b, k, q, t=1}+s b k_{b, k, t=1}, \forall b \in B, k \in K
\end{aligned}
$$


Notably, the parameter $\varepsilon_{b, t}$ captures the deterioration feature of poultry waste ${ }^{34,35}$. In addition, a "cyclic" manner of inventory balance is considered and shown in constraint (5) that the poultry litter storage amount at the beginning of the year and the inventory level at the end of the year are the same.

Likewise, the mass balance of products in pyrolysis facilities are shown in constraints (6) and (7). Set of demand zones are denoted as $D$.

$$
\begin{aligned}
& \sum_{q} w p k_{k, p, q, t}+s p k_{k, p, t-1}=\sum_{d} \sum_{m} f k d_{d, k, p, m, t}+s p k_{k, p, t}, \forall k \in K, p \in P, t \geq 2 \\
& \sum_{q} w p k_{k, p, q, t=1}+s p k_{k, p, t=T \mid}=\sum_{d} \sum_{m} f k d_{d, k, p, m, t=1}+s p k_{k, p, t=1}, \forall k \in K, p \in P
\end{aligned}
$$

where $s p k_{k, p, t}$ is the storage level of product type $p$ at pyrolysis facility $k$ in time period $t$, and

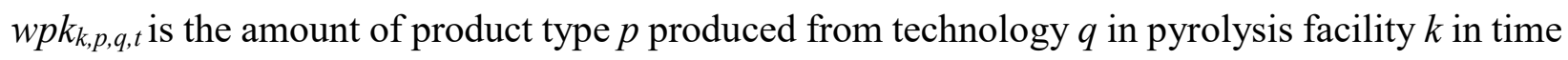
period $t$. $f k d_{d, k, p, m, t}$ denotes the amount of product type $p$ shipped from pyrolysis facility $k$ to demand zone $d$ using transportation mode $m$ in time period $t$.

The mass balance of intermediates such as bio-oil and syngas are derived in a similar manner in constraints (8) and (9). Since some intermediates are not suitable for further upgrading from an economic or environmental perspective, they are processed on-site at the pyrolysis facilities, and the processed amount of intermediate $g$ in pyrolysis facility $k$ in time period $t$ is presented by $r g k_{g, k, t}$. Set of upgrading facilities indexed by $l$ is denoted as $L$.

$$
\begin{aligned}
& \sum_{q} w g k_{g, k, q, t}+s g k_{g, k, t-1}=\sum_{l} \sum_{m} f k l_{g, k, l, m, t}+s g k_{g, k, t}+r g k_{g, k, t}, \forall g \in G, k \in K, t \geq 2 \\
& \sum_{q} w g k_{g, k, q, t=1}+s g k_{g, k, t=|T|}=\sum_{l} \sum_{m} f k l_{g, k, l, m, t=1}+s g k_{g, k, t=1}+r g k_{g, k, t=1}, \forall g \in G, k \in K
\end{aligned}
$$

where $s g k_{g, k, t}$ is the storage level of intermediate type $g$ at pyrolysis facility $k$ in time period $t$, and $w g k_{g, k, q, t}$ is the amount of intermediate type $g$ produced through technology $q$ in pyrolysis facility $k$ in time period $t . f k l_{g, k, l, m, t}$ denotes the amount of bio-oil or biogas type $g$ shipped from pyrolysis facility $k$ to upgrading facility $l$ using transportation mode $m$ in time period $t$.

Constraint (10) shows the calculation of the processed amount of bio-oil or biogas at pyrolysis facilities. Binary 0-1 parameter $Y_{k, g}$ equals to 1 for biogas type $g$ which is processed in pyrolysis facility $k$, and $Y_{k, g}$ equals to 0 for bio-oil type $g$ since it is not processed locally at pyrolysis facilities.

$$
r g k_{g, k, t}=\sum_{q} Y_{k, g} \cdot w g k_{g, k, q, t}, \forall g \in G, k \in K, t \in T
$$


Constraint (11) indicates that at most one pyrolysis technology and capacity level can be selected for a given pyrolysis facility. Set $R$ represents capacity levels of facilities, and binary variable $x_{k, q, r}$ indicates whether pyrolysis technology $q$ and capacity level $r$ are selected for pyrolysis facility $k$. We note that if the left-hand side of constraint (11) equals to zero, no pyrolysis facilities will be built at location $k$.

$$
\sum_{q} \sum_{r} x_{k, q, r} \leq 1, \forall k \in K
$$

Constraint (12) enforces that for each technology $q$, the number of corresponding pyrolysis facilities has an upper bound $N K_{q}$.

$$
\sum_{k} \sum_{r} x_{k, q, r} \leq N K_{q}, \forall q \in Q
$$

The capacity range of a pyrolysis facility is given in constraint (13). capk $k_{k, q, r}$ is the annual production capacity of pyrolysis facility $k$ with pyrolysis technology $q$ and capacity level $r$, and $P R K_{k, q, r}$ is the upper bound of the capacity (in terms of dry tons of standard biomass) of pyrolysis facility $k$ with technology $q$ and capacity level $r$.

$$
P R K_{k, q, r-1} x_{k, q, r} \leq \operatorname{capk}_{k, q, r} \leq P R K_{k, q, r} x_{k, q, r}, \forall k \in K, q \in Q, r \in R
$$

Constraint (14) calculates the capital cost of pyrolysis facilities. Due to the scaling effect of facility economics, the capital cost of a pyrolysis facility $k$ (tcapk $k_{k}$ ) is represented by an interpolated piecewise linear function ${ }^{17,36}$ based on different capacity levels. $C R K_{k, q, r}$ denotes the capital cost of pyrolysis facility $k$ with technology $q$ at capacity level $r$.

$$
\operatorname{tcapk}_{k}=\sum_{q} \sum_{r}\left[C R K_{k, q, r-1} x_{k, q, r}+\left(\operatorname{capk}_{k, q, r}-P R K_{k, q, r-1} x_{k, q, r}\right)\left(\frac{C R K_{k, q, r}-C R K_{k, q, r-1}}{P R K_{k, q, r}-P R K_{k, q, r-1}}\right)\right], \forall k \in K
$$

Likewise, the annual fixed O\&M cost $\left(t c f p k_{k}\right)$ can be calculated through constraint (15). $C F K_{k, q}$ is a proportion of capital cost that serves as the rate of annual fixed O\&M cost. Notably, the cost rate $C F K_{k, q}$ may vary according to different pyrolysis technology $q$ and facility location $k$.

$$
\begin{aligned}
& t c f p k_{k}= \\
& \sum_{q}\left\{C F K_{k, q} \sum_{r}\left[C R K_{k, q, r-1} x_{k, q, r}+\left(c a p k_{k, q, r}-P R K_{k, q, r-1} x_{k, q, r}\right)\left(\frac{C R K_{k, q, r}-C R K_{k, q, r-1}}{P R K_{k, q, r}-P R K_{k, q, r-1}}\right)\right]\right\}, \forall k \in K
\end{aligned}
$$


Constraints (16) and (17) represent the government incentive in the form of investment refund $\left(i_{c k} k_{k}\right)$. Owing to the environmental benefits of sustainable products from similar processes, government incentives are available in certain regions. The government would refund the pyrolysis facilities with either a proportion of their capital cost (where the refund proportion is INCP) or a maximum incentive amount (INCM), whichever is smaller. Clearly, no incentive will be granted if no pyrolysis facility is built in location $k$.

$$
\begin{aligned}
& \text { inck }_{k} \leq I N C M \sum_{q} \sum_{r} x_{k, q, r}, \forall k \in K \\
& \text { inck }_{k} \leq I N C P \cdot \text { tcapk }_{k}, \forall k \in K
\end{aligned}
$$

Constraints (18) and (19) calculate the annual cost $\left(r c v p k_{k}\right)$ and environmental impact (revpkk) associated with intermediate processing in pyrolysis facility $k$, respectively.

$$
\begin{aligned}
& r c v p k_{k}=\sum_{g} C N O K_{k, g} \cdot w g k_{g, k, t}, \forall k \in K \\
& r e v p k_{k}=\sum_{g} E N O K_{k, g} \cdot w g k_{g, k, t}, \forall k \in K
\end{aligned}
$$

where $C N O K_{k, g}$ and $E N O K_{k, g}$ are unit cost and unit emissions of processing intermediate type $g$ in pyrolysis facility $k$, respectively.

Constraint (20) indicates the range of organic waste processed amount in pyrolysis facilities. The total litter consumption amount in pyrolysis facility $k$ in time period $t$ using technology $q$ ( $\left.\sum_{b} \rho_{b} w b k_{b, k, q, t}\right)$ is allowed to vary between the lower bound $\left(\theta_{k, q} \frac{H_{t}}{H Y} \sum_{r} c a p k_{k, q, r}\right)$ and the corresponding capacity $\left(\frac{H_{t}}{H Y} \sum_{r} c a p k_{k, q, r}\right)$.

$$
\theta_{k, q} \frac{H_{t}}{H Y} \sum_{r} \operatorname{capk}_{k, q, r} \leq \sum_{b} \rho_{b} w b k_{b, k, q, t} \leq \frac{H_{t}}{H Y} \sum_{r} \operatorname{capk}_{k, q, r}, \forall k \in K, q \in Q, t \in T
$$

where $\theta_{k, q}$ is the minimum capacity utilization proportion for pyrolysis facility $k$ and technology $q$. $H_{t}$ is the duration of a time period $t$, and $H Y$ is the duration time of a year. $\rho_{b}$ is the mass quantity of standard dry biomass of 1 dry ton of poultry waste type $b$.

Constraints (21) and (22) represent the production scale of products and intermediates from the pyrolysis process. $\alpha_{b, p, q}$ and $\beta_{b, g, q}$ are conversion rates for product $p$ and intermediate $g$, respectively, from poultry waste type $b$ using pyrolysis technology $q$. 


$$
\begin{aligned}
& w p k_{k, p, q, t}=\sum_{b} \alpha_{b, p, q} w b k_{b, k, q, t}, \forall k \in K, p \in P, q \in Q, t \in T \\
& w g k_{g, k, q, t}=\sum_{b} \beta_{b, g, q} w b k_{b, k, q, t}, \forall g \in G, k \in K, q \in Q, t \in T
\end{aligned}
$$

Constraint (23) describes the storage of poultry waste feedstock. The inventory level of feedstock $\left(s b k_{b, k, t}\right)$ should be higher than the safety storage level, which is represented by the righthand side, to avoid unexpected circumstances. $S K_{k, t}$ represents the safety period for pyrolysis facility $k$ in time period $t$, and the safety storage level is defined as the amount of processed feedstock within the safety period.

$$
s b k_{b, k, t} \geq \frac{S K_{k, t}}{H_{t}} \sum_{q} w b k_{b, k, q, t}, \forall b \in B, k \in K, t \in T
$$

\section{Intermediate upgrading facilities}

Constraints (24) and (25) represent the mass balance of intermediate feedstock in upgrading facilities. The set of upgrading technologies indexed by $q$ ' are included in technology set $Q$.

$$
\begin{aligned}
& \sum_{k} \sum_{m} f k l_{g, k, l, m, t}+s g l_{g, l, t-1}=\sum_{q^{\prime}} w g l_{g, l, q^{\prime}, t}+s g l_{g, l, t}, \forall g \in G, l \in L, t \geq 2 \\
& \sum_{k} \sum_{m} f k l_{g, k, l, m, t=1}+s g l_{g, l, t=T \mid}=\sum_{q^{\prime}} w g l_{g, l, q^{\prime}, t=1}+s g l_{g, l, t=1}, \forall g \in G, l \in L
\end{aligned}
$$

where $s g l_{g, l, t}$ is the storage level of intermediate feedstock $g$ at upgrading facility $l$ in time period $t$, and $w g l_{g, l, q}{ }^{\prime}, t$ represents the consumption of intermediate type $g$ processed through technology $q^{\prime}$ at upgrading facility $l$ in time period $t$.

Likewise, the mass balance of liquid fuel products at upgrading facilities is shown in constraints (26) and (27).

$$
\begin{aligned}
& \sum_{q^{\prime}} w p l_{l, p, q^{\prime}, t}+s p l_{l, p, t-1}=\sum_{d} \sum_{m} f l d_{d, l, p, m, t}+s p l_{l, p, t}, \forall l \in L, p \in P, t \geq 2 \\
& \sum_{q^{\prime}} w p l_{l, p, q^{\prime}, t=1}+s p l_{l, p, t=|T|}=\sum_{d} \sum_{m} f l d_{d, l, p, m, t=1}+s p l_{l, p, t=1}, \forall l \in L, p \in P
\end{aligned}
$$

where $w p l_{l, p, q^{\prime}, t}$ is the amount of liquid fuel product type $p$ produced from technology $q^{\prime}$ at upgrading facility $l$ in time period $t$, and the transportation of liquid fuel product $p$ shipped from upgrading facility $l$ to demand zone $d$ using transportation mode $m$ in time period $t$ is denoted as fld $d, l, p, m, t$. 
Constraint (28) shows that at most one upgrading technology and capacity level can be selected for an upgrading facility. Binary variable $z_{l, q}{ }^{\prime}, r$ indicates whether upgrading technology $q$, and capacity level $r$ is selected for upgrading facility $l$.

$\sum_{q^{\prime}} \sum_{r} z_{l, q^{\prime}, r} \leq 1, \forall l \in L$

In constraint (29), $N L_{q}$, represents the maximum number of upgrading facilities that can be built with technology $q$ '.

$$
\sum_{l} \sum_{r} z_{l, q^{\prime}, r} \leq N L_{q^{\prime}}, \forall q^{\prime} \in Q
$$

Constraint (30) gives the capacity range of an upgrading facility. capl $_{l, q^{\prime}, r}$ and $P R L_{l, q^{\prime}, r}$ are the annual production capacity (in terms of gallons of gasoline equivalent (GEG)) and the upper bound of the capacity, respectively, for upgrading facility $l$ with technology $q$ ' and capacity level $r$.

$$
P R L_{l, q^{\prime}, r-1} z_{l, q^{\prime}, r} \leq \operatorname{capl}_{l, q^{\prime}, r} \leq P R L_{l, q^{\prime}, r} z_{l, q^{\prime}, r}, \forall l \in L, q^{\prime} \in Q, r \in R
$$

Constraint (31) indicates the maximum transportation distance $\left(M D S_{g, m, t}\right)$ of intermediate feedstock. Due to stability concerns of intermediate feedstock such as bio-oil, we consider that no intermediates are transported from pyrolysis facility $k$ to upgrading facility $l$, if the distance between the two facilities $\left(D S K L_{k, l, m}\right)$ exceeds the maximum allowable transportation distance $\left(M D S_{g, m, t}\right)$.

$$
f k l_{g, k, l, m, t}=0, \forall(g, k, l, m, t) \mid\left(M D S_{g, m, t} \leq D S K L_{k, l, m}\right)
$$

Constraints (32) and (33) calculate the capital cost (tcapl $)$ and the annual fixed O\&M cost $\left(t c f p l_{l}\right)$ of an upgrading facility $l$, respectively. $C R L_{l, q^{\prime}, r}$ denotes the capital cost of upgrading facility $l$ with technology $q$ ' at capacity level $r$, and $C F L_{l, q}$, is a proportion of capital investment that serves as the annual fixed O\&M cost.

$$
\begin{aligned}
& \operatorname{tcapl}_{l}=\sum_{q^{\prime}} \sum_{r}\left[C R L_{l, q^{\prime}, r-1} z_{l, q^{\prime}, r}+\left(\operatorname{capl}_{l, q^{\prime}, r}-P R L_{l, q^{\prime}, r-1} z_{l, q^{\prime}, r}\right)\left(\frac{C R L_{l, q^{\prime}, r}-C R L_{l, q^{\prime}, r-1}}{P R L_{l, q^{\prime}, r}-P R L_{l, q^{\prime}, r-1}}\right)\right], \forall l \in L \\
& \operatorname{tcfpl} l_{l}=\sum_{q^{\prime}}\left\{C F L_{l, q^{\prime}} \sum_{r}\left[C R L_{l, q^{\prime}, r-1} z_{l, q^{\prime}, r}+\left(\operatorname{capl}_{l, q^{\prime}, r}-P R L_{l, q^{\prime}, r-1} z_{l, q^{\prime}, r}\right)\left(\frac{C R L_{l, q^{\prime}, r}-C R L_{l, q^{\prime}, r-1}}{P R L_{l, q^{\prime}, r}-P R L_{l, q^{\prime}, r-1}}\right)\right]\right\}, \forall l \in L
\end{aligned}
$$

Constraints (34) and (35) calculate the government incentive in the form of investment refund 
$(i n c l)$ for the upgrading facilities. The amount of refund would be either a proportion (INCP) of the capital cost or a maximum incentive amount (INCM), whichever is smaller.

$$
\begin{aligned}
& \text { incl }_{l} \leq I N C M \sum_{q^{\prime}} \sum_{r} z_{l, q^{\prime}, r}, \forall l \in L \\
& \text { incl }_{l} \leq I N C P \cdot \text { tcapl }_{l}, \forall l \in L
\end{aligned}
$$

Constraint (36) gives the range of liquid fuel production amount in the upgrading facilities. The total production level of liquid fuel in terms of GEG $\left(\sum_{p} \varphi_{p} w p l_{l, p, q^{\prime}, t}\right)$ should be higher than the minimum production level $\left(\eta_{l, q^{\prime}} \frac{H_{t}}{H Y} \sum_{r} \operatorname{capl}_{l, q^{\prime}, r}\right)$, and should not exceed the facility capacity

$$
\begin{aligned}
& \left(\frac{H_{t}}{H Y} \sum_{r} \operatorname{capl}_{l, q^{\prime}, r}\right) . \\
& \quad \eta_{l, q^{\prime}} \frac{H_{t}}{H Y} \sum_{r} \operatorname{capl}_{l, q^{\prime}, r} \leq \sum_{p} \varphi_{p} w p l_{l, p, q^{\prime}, t} \leq \frac{H_{t}}{H Y} \sum_{r} \operatorname{capl}_{l, q^{\prime}, r}, \forall l \in L, q^{\prime} \in Q, t \in T
\end{aligned}
$$

where $\eta_{l, q}$, is the minimum capacity utilization proportion for upgrading facility $l$ with technology $q^{\prime}$, and $\varphi_{p}$ is the GEG of 1 gallon of product $p$.

Constraint (37) represents the mass balance for the production of liquid fuel from the upgrading process. $\gamma_{g, p, q}$ is the conversion rate of fuel product $p$ from intermediate feedstock $g$ using upgrading technology $q$ '.

$$
w p l_{l, p, q^{\prime}, t}=\sum_{g} \gamma_{g, p, q^{\prime}} w g l_{g, l, q^{\prime}, t}, \forall l \in L, p \in P, q^{\prime} \in Q, t \in T
$$

Constraint (38) indicates the storage of intermediate feedstock in upgrading facilities. The inventory level of intermediate $\left(s g l_{g, l, t}\right)$ should be higher than the safety storage level represented by the right-hand side.

$$
s g l_{g, l, t} \geq \frac{S L_{l, t}}{H_{t}} \sum_{q^{\prime}} w g l_{g, l, q^{\prime}, t}, \forall g \in G, l \in L, t \in T
$$

where $S L_{l, t}$ represents the safety period for upgrading facility $l$ in time period $t$.

\section{Product distribution system}

Constraint (39) calculates the sales of product $p$ in demand zone $d$ in time period $t\left(\operatorname{sold}_{d, p, t}\right)$, 
which equals to the total amount of this product shipped from pyrolysis facilities $\left(\sum_{k} \sum_{m} f k d_{d, k, p, m, t}\right)$ and upgrading facilities $\left(\sum_{l} \sum_{m} f l d_{d, l, p, m, t}\right)$ in the time period with all types of transportation modes.

$$
\sum_{k} \sum_{m} f k d_{d, k, p, m, t}+\sum_{l} \sum_{m} f l d_{d, l, p, m, t}=\operatorname{sold}_{d, p, t}, \forall d \in D, p \in P, t \in T
$$

Constraint (40) shows the feasible ranges for the product sales. $D E M_{d, p, t}^{U}$ is the upper bound of demand of product type $p$ in demand zone $d$ in time period $t$, and $D E M_{d, p, t}^{L}$ is the lower bound of demand that should be fulfilled by the sustainable products.

$$
D E M_{d, p, t}^{L} \leq \operatorname{sold}_{d, p, t} \leq D E M_{d, p, t}^{U}, \forall d \in D, p \in P, t \in T
$$

\section{Objective functions}

\section{Economic objective function}

The economic objective is to maximize the unit annualized profit based on techno-economic analysis (TEA). Total annualized profit accounts for total annual revenue, total annualized capital cost, total annual operation cost, and total annualized government incentive. Total amount based on the functional unit can be determined by the amount of poultry litter consumption.

The total amount of annual revenue is calculated through eq. (41), which consists of the sales revenue of all products in all demand zones through all time periods in a year. $P R I_{d, p, t}$ denotes the price of product $p$ at demand zone $d$ in time period $t$.

$$
R E V=\sum_{d} \sum_{p} \sum_{t} P R I_{d, p, t} \cdot \text { sold }_{d, p, t}
$$

The total annualized capital cost is calculated through eq. (42) using the equivalent annual cost method, and it accounts for the total annualized capital investment of both pyrolysis facilities and upgrading facilities. Notably, the time value of money has been considered in this study, by including the annual discount rate $(I R)$ and the lifetime of facilities $(N Y)$ in the equivalent annual cost method.

$$
C_{\text {capital }}=\frac{I R(1+I R)^{N Y}}{(1+I R)^{N Y}-1}\left(\sum_{k} \text { tcapk }_{k}+\sum_{l} \text { tcapl }_{l}\right)
$$

The total annual operation cost is calculated from eqs (43) - (47), including feedstock acquisition cost, product distribution cost, production cost for pyrolysis facilities and upgrading 
facilities, transportation cost and storage cost of poultry waste feedstock, intermediates and products. $C B M_{b, i, t}$ is the unit acquisition cost of feedstock $b$ at poultry farm $i$ in time period $t$, and $C L D_{d, p}$ is the unit distribution cost of product $p$ at demand zone $d$. To calculate the production cost, both fixed and variable production costs are considered. The fixed production cost is a proportion of total capital cost, and the variable production cost depends on the conversion amount of feedstock, intermediate and products. The transportation cost consists of distance-fixed transportation cost and distance-variable transportation cost. $D F C B_{b, m}, D F C G_{g, m}$ and $D F C P_{m, p}$ are the distance-fixed cost with transportation mode $m$ for feedstock type $b$, intermediate type $g$ and product type $p$, respectively. Likewise, $D V C B_{b, m}, D V C G_{g, m}$ and $D V C P_{m, p}$ are the distance-variable cost with transportation mode $m$ for feedstock type $b$, intermediate type $g$ and product type $p$, respectively. The unit inventory holding costs are denoted as $H B K_{b, k, t}, H G K_{g, k, t}, H G L_{g, l, t}, H P K_{k, p, t}$ and $H P L_{l, p, t}$.

$$
\begin{aligned}
C_{\text {acquisition }}= & \sum_{b} \sum_{i} \sum_{t} C B M_{b, i, t} \cdot \operatorname{bmp}_{b, i, t} \\
C_{\text {distribution }}= & \sum_{d} \sum_{p} \sum_{t} C L D_{d, p} \cdot \operatorname{sold}_{d, p, t} \\
C_{\text {production }}= & \sum_{k} t c f p k_{k}+\sum_{l} t c f p l_{l} \\
& +\sum_{k} \sum_{b} \sum_{q} \sum_{t} C P K_{k, q} \rho_{b} w b k_{b, k, q, t}+\sum_{k} r c v p k_{k} \\
& +\sum_{l} \sum_{p} \sum_{q^{\prime}} \sum_{t} C P L_{l, q^{\prime}} \varphi_{p} w p l_{l, p, q^{\prime}, t} \\
= & \sum_{b} \sum_{i} \sum_{k} \sum_{m} \sum_{t}\left(D F C B_{b, m}+D V C B_{b, m} D S I K_{i, k, m}\right) f i k_{b, i, k, m, t} \\
& +\sum_{g} \sum_{k} \sum_{l} \sum_{m} \sum_{t}\left(D F C G_{g, m}+D V C G_{g, m} D S K L_{k, l, m}\right) f k l_{g, k, l, m, t} \\
& +\sum_{d} \sum_{k} \sum_{p} \sum_{m} \sum_{t}\left(D F C P_{m, p}+D V C P_{m, p} D S K D_{d, k, m}\right) f k d_{d, k, p, m, t} \\
& +\sum_{d} \sum_{l} \sum_{p} \sum_{m} \sum_{t}\left(D F C P_{m, p}+D V C P_{m, p} D S L D_{d, l, m}\right) f l d_{d, l, p, m, t}
\end{aligned}
$$




$$
\begin{aligned}
C_{\text {storage }}= & \sum_{b} \sum_{k} \sum_{t} H_{t} \cdot H B K_{b, k, t} \cdot s b k_{b, k, t} \\
& +\sum_{g} \sum_{k} \sum_{t} H_{t} \cdot H G K_{g, k, t} \cdot s g k_{g, k, t} \\
& +\sum_{k} \sum_{p} \sum_{t} H_{t} \cdot H P K_{k, p, t} \cdot s p k_{k, p, t} \\
& +\sum_{g} \sum_{l} \sum_{t} H_{t} \cdot H G L_{g, l, t} \cdot s g l_{g, l, t} \\
& +\sum_{l} \sum_{p} \sum_{t} H_{t} \cdot H P L_{l, p, t} \cdot s p l_{l, p, t}
\end{aligned}
$$

As shown in eq. (48), the total annualized government incentive includes both annualized construction incentives $\left(\frac{I R(1+I R)^{N Y}}{(1+I R)^{N Y}-1}\left(\sum_{k} i n c k_{k}+\sum_{l} i n c l_{l}\right)\right)$ and volumetric incentives on biofuel consumption $\left(\sum_{d} \sum_{p} \sum_{t} I N C V_{d, p} \cdot \operatorname{sold}_{d, p, t}\right) . I N C V_{d, p}$ is the volumetric production incentive of sustainable product type $p$ sold to demand zone $d$.

$$
C_{\text {incentive }}=\frac{I R(1+I R)^{N Y}}{(1+I R)^{N Y}-1}\left(\sum_{k} \text { inck }_{k}+\sum_{l} \text { incl }_{l}\right)+\sum_{d} \sum_{p} \sum_{t} I N C V_{d, p} \cdot \operatorname{sold}_{d, p, t}
$$

The economic objective function, to maximize the unit annualized profit, is shown in eq. (49).

$$
\max u p=\frac{R E V-C_{\text {capital }}-C_{\text {acquisition }}-C_{\text {distribution }}-C_{\text {production }}-C_{\text {transportation }}-C_{\text {storage }}+C_{\text {incentive }}}{\sum_{b} \sum_{i} \sum_{t} \rho_{b} b m p_{b, i, t}}
$$

\section{Environmental objective function}

The environmental objective function is to minimize the unit annual $\mathrm{CO}_{2}$-eq $\mathrm{GHG}$ emissions from the entire poultry waste supply chain network. To formulate the environmental objective function, we apply "cradle-to-gate" life cycle analysis, which includes emissions from the following life cycle stages:

- feedstock acquisition, transportation and storage;

- conversion of feedstock to intermediates and products;

- intermediate transportation, upgrading and storage;

- conversion of intermediates to products;

- product transportation, distribution and usage.

As shown in eqs (50) - (55), the net $\mathrm{CO}_{2}$-eq GHG emissions take into account emissions 
(carbon dioxide, nitrous oxide, methane) introduced by feedstock acquisition, product distribution, pyrolysis processes, upgrading processes, transportation and storage, as well as carbon dioxide sequestration from produced biochar.

$$
\begin{aligned}
& E_{\text {acquisition }}=\sum_{b} \sum_{i} \sum_{t} E B M_{b, i, t} \cdot b m p_{b, i, t} \\
& E_{\text {distribution }}=\sum_{d} \sum_{p} \sum_{t} E L D_{d, p} \cdot \operatorname{sold}_{d, p, t} \\
& E_{\text {production }}=\sum_{k} \sum_{b} \sum_{q} \sum_{t} E P K_{k, q} \rho_{b} w b k_{b, k, q, t}+\sum_{k} \text { revpk }_{k} \\
& +\sum_{l} \sum_{p} \sum_{q^{\prime}} \sum_{t} E P L_{l, q^{\prime}} \varphi_{p} w p l_{l, p, q^{\prime}, t} \\
& E_{\text {transportation }}=\sum_{b} \sum_{i} \sum_{k} \sum_{m} \sum_{t}\left(\operatorname{ETRB}_{b, m} D S I K_{i, k, m}\right) f i k_{b, i, k, m, t} \\
& +\sum_{g} \sum_{k} \sum_{l} \sum_{m} \sum_{t}\left(E T R G_{g, m} D S K L_{k, l, m}\right) f k l_{g, k, l, m, t} \\
& +\sum_{d} \sum_{k} \sum_{p} \sum_{m} \sum_{t}\left(E T R P_{m, p} D S K D_{d, k, m}\right) f k d_{d, k, p, m, t} \\
& +\sum_{d} \sum_{l} \sum_{p} \sum_{m} \sum_{t}\left(E T R P_{m, p} D S L D_{d, l, m}\right) f l d_{d, l, p, m, t} \\
& E_{\text {storage }}=\sum_{b} \sum_{k} \sum_{t} H_{t} \cdot E H B_{b, t} \cdot s b k_{b, k, t} \\
& +\sum_{g} \sum_{k} \sum_{t} H_{t} \cdot E H G_{g, t} \cdot s g k_{g, k, t} \\
& +\sum_{k} \sum_{p} \sum_{t} H_{t} \cdot \mathrm{EHP}_{p, t} \cdot s p k_{k, p, t} \\
& +\sum_{g} \sum_{l} \sum_{t} H_{t} \cdot E H G_{g, t} \cdot s g l_{g, l, t} \\
& +\sum_{l} \sum_{p} \sum_{t} H_{t} \cdot \operatorname{EHP}_{p, t} \cdot \operatorname{spl}_{l, p, t} \\
& E_{\text {sequestration }}=\sum_{b} \sum_{i} \sum_{t} \operatorname{ESCB}_{b} \cdot b m p_{b, i, t}+\sum_{d} \sum_{p} \sum_{t} \operatorname{ESCP}_{p} \cdot \operatorname{sold}_{d, p, t}
\end{aligned}
$$

where $E B M_{b, i, t}$ is the unit emissions introduced by the acquisition of poultry waste $b$ at poultry farm $i$ in time period $t$, and $E L D_{d, p}$ is the unit emissions associated with the distribution of product $p$ at demand zone $d . E P K_{k, q}$ and $E P L_{l, q}$, are the unit production emissions of pyrolysis facility $k$ with technology $q$ and upgrading facility $l$ with technology $q^{\prime}$. ETR $B_{b, m}, E T R G_{g, m}$ and $E T R P_{m, p}$ are the unit emissions introduced by transportation using transportation mode $m$ for feedstock type $b$, intermediate type $g$ and product type $p$, respectively. The unit emissions of inventory storage are 
denoted as $E H B_{b, t}, E H G_{g, t}$ and $E H P_{p, t}$. The unit carbon dioxide sequestration due to feedstock type $b$ and product type $p$ are represented by $E S C B_{b}$ and $E S C P_{p}$, respectively.

The environmental objective of minimizing unit annual $\mathrm{CO}_{2}$-eq $\mathrm{GHG}$ emission is shown in eq (56).

$$
\min u e=\frac{E_{\text {acquisition }}+E_{\text {distribution }}+E_{\text {production }}+E_{\text {transportation }}+E_{\text {storage }}-E_{\text {sequestration }}}{\sum_{b} \sum_{i} \sum_{t} \rho_{b} b m p_{b, i, t}}
$$

\section{Multi-objective MILFP model}

The multi-period poultry waste supply chain optimization problem can be formulated as a multi-objective MILFP model denoted as (P0). The outline of (P0) is shown as follows:

(P0) max Unit Annualized Profit given by (49)

min Unit Annual $\mathrm{CO}_{2}$-eq GHG Emissions given by (56)

s.t. Poultry litter feedstock supply system constraints: (1) - (3)

Pyrolysis facility constraints: (4) - (23)

Upgrading facility constraints: (24) - (38)

Product distribution system constraints: (39) - (40)

Economic constraints: (41) - (48)

Environmental constraints: (50) - (55)

The model (P0) is a multi-objective MILFP model, where two fractional objective functions are involved. The denominators and numerators of both objective functions are linear, and all constraints are in linear forms. 


\section{Nomenclature}

\section{Sets/indices}

$B \quad$ set of poultry litter feedstocks indexed by $b$

$D \quad$ set of demand zones indexed by $d$

$G \quad$ set of intermediate products indexed by $g$

$I \quad$ set of harvesting sites indexed by $i$

$K \quad$ set of pyrolysis facilities indexed by $k$

$L \quad$ set of intermediate upgrading facilities indexed by $l$

$M \quad$ set of transportation modes indexed by $m$

$P \quad$ set of final products indexed by $p$

$Q \quad$ set of pyrolysis technologies (indexed by q) and upgrading technologies (indexed by $\left.q^{\prime}\right)$

$R \quad$ set of capacity levels of facilities indexed by $r$

$T \quad$ set of time periods indexed by $t$

\section{Parameters}

$B A_{b, i, t} \quad$ available amount of poultry waste type $b$ in harvesting site $i$ at time period $t$

$C B M_{b, i, t} \quad$ farm-gate cost of poultry litter feedstock type $b$ from harvesting site $i$ at time $t$

$C F K_{k, q} \quad$ fixed annual O\&M cost as the proportion of the total investment cost of pyrolysis facility $k$ with technology $q$

$C F L_{l, q}$ fixed annual O\&M cost as the proportion of the total investment cost of upgrading facility $l$ with technology $q$,

$C L D_{d, p} \quad$ local distribution cost of unit quantity of product $p$ at demand zone $d$

$C N O K_{k, g} \quad$ net unit processing cost on intermediate type $g$ in pyrolysis facility $k$

$C P K_{k, q} \quad$ net unit production cost per dry ton of standard biomass in pyrolysis facility $k$ with technology $q$ (after considering charcoal credit)

$C P L_{l, q}$, net unit production cost per gallon of gasoline equivalent of liquid transportation fuel in upgrading facility $l$ with technology $q^{\prime}$ (after considering charcoal credit)

$C R K_{k, q, r} \quad$ total capital investment of pyrolysis facility $k$ with technology $q$ and capacity level $r$ 
$C R L_{l, q^{\prime}, r} \quad$ total capital investment of upgrading facility $l$ with technology $q$ ' and capacity level $r$

$D E M_{d, p, t}^{L} \quad$ lower bound of the demand for product $p$ at demand zone $d$ at time $t$

$D E M_{d, p, t}^{U} \quad$ upper bound of the demand for product $p$ at demand zone $d$ at time $t$

$D F C B_{b, m} \quad$ distance fixed cost of poultry waste type $b$ with transportation mode $m$

$D F C G_{g, m} \quad$ distance fixed cost of intermediate type $g$ with transportation mode $m$

$D F C P_{m, p} \quad$ distance fixed cost of product type $p$ with transportation mode $m$

$D S I K_{i, k, m} \quad$ distance from harvesting site $i$ to pyrolysis facility $k$ with transportation mode $m$

$D S K D_{d, k, m} \quad$ distance from pyrolysis facility $k$ to demand zone $d$ with transportation mode $m$

$D S K L_{k, l, m} \quad$ distance from pyrolysis facility $k$ to intermediate upgrading facility $l$ with transportation mode $m$

$D S L D_{d, l, m} \quad$ distance from upgrading facility $l$ to demand zone $d$ with transportation mode $m$

$D V C B_{b, m} \quad$ distance variable cost of poultry waste type $b$ with transportation mode $m$

$D V C G_{g, m} \quad$ distance variable cost of intermediate type $g$ with transportation mode $m$

$D V C P_{m, p} \quad$ distance variable cost of product type $p$ with transportation mode $m$

$E B M_{b, i, t} \quad$ emission due to cultivation and acquisition of unit quantity of litter feedstock type $b$ from harvesting site $i$ at time $t$

$E H B_{b, t} \quad$ unit emission of storing unit quantity of poultry waste type $b$ at time period $t$

$E H G_{g, t} \quad$ unit emission of storing unit quantity of intermediate type $g$ at time period $t$

$E H P_{p, t} \quad$ unit emission of storing unit quantity of product type $p$ at time period $t$

$E L D_{d, p} \quad$ emission due to local distribution of product type $p$ at demand zone $d$

$E N O K_{k, g} \quad$ net unit emission from processing intermediate type $g$ in pyrolysis facility $k$

$E P K_{k, q} \quad$ emission of processing a dry ton of standard biomass in pyrolysis facility $k$ with technology $q$

$E P L_{l, q^{\prime}} \quad$ emission of producing a gallon of gasoline equivalent of fuel product in upgrading facility $l$ with technology $q^{\prime}$

$E S C B_{b} \quad$ emission credit of soil carbon dioxide sequestration due to poultry waste type $b$

$E S C P_{p} \quad$ emission credit of soil carbon dioxide sequestration due to product type $p$ (mainly biochar)

$E T R B_{b, m} \quad$ emission of transporting unit amount of poultry waste type $b$ for unit distance with transportation mode $m$ 
$E T R G_{g, m} \quad$ emission of transporting unit amount of intermediate type $g$ for unit distance with transportation mode $m$

$E T R P_{m, p} \quad$ emission of transporting unit amount of product $p$ for unit distance with transportation mode $m$

$H_{t} \quad$ duration of time period $t$

$H B K_{b, k, t} \quad$ unit inventory holding cost of poultry waste type $b$ in pyrolysis facility $k$ at time $t$

$H G K_{g, k, t} \quad$ unit inventory holding cost of intermediate type $g$ in pyrolysis facility $k$ at time $t$

$H G L_{g, l, t} \quad$ unit inventory holding cost of intermediate type $g$ in upgrading facility $l$ at time $t$

$H P K_{k, p, t} \quad$ unit inventory holding cost of product type $p$ in pyrolysis facility $k$ at time $t$

$H P L_{l, p, t} \quad$ unit inventory holding cost of product type $p$ in upgrading facility $l$ at time $t$

HY production time duration of a year

INCM maximum incentive that can be provided for the construction of organic waste conversion facilities

INCP maximum proportion of construction cost of organic waste conversion facilities that can be covered by government incentive

$I N C V_{d, p} \quad$ volumetric production incentive of product type $p$ sold to demand zone $d$

IR discount rate

$M C_{b} \quad$ moisture content (in weight) of poultry waste type $b$

$M D S_{g, m, t} \quad$ maximum allowable transportation distance (due to the stability) of intermediate type $g$ with transportation mode $m$ at time period $t$

$N K_{q} \quad$ maximum number of pyrolysis facilities with technology $q$ that can be constructed

$N L_{q}$, maximum number of upgrading facilities with technology $q$ ' that can be constructed

$N Y \quad$ project lifetime in terms of years

$P R I_{d, p, t} \quad$ price of product type $p$ at demand zone $d$ in time period $t$

$P R K_{k, q, r} \quad$ upper bound of the capacity (in terms of dry tons of standard biomass) of pyrolysis facility $k$ with technology $q$ and capacity level $r$

$P R L_{l, q^{\prime}, r} \quad$ upper bound of the capacity (in terms of gallons of gasoline equivalent) of upgrading facility $l$ with technology $q$ ' and capacity level $r$

$S K_{k, t} \quad$ safety stock inventory that should be hold to cover the production shortage in pyrolysis facility $k$ at time $t$ 
$S L_{l, t} \quad$ safety stock inventory that should be hold to cover the production shortage in upgrading facility $l$ at time $t$

UPGK upper bound for the amount of intermediate production

$W C I K_{i, k, m, t}$ weight capacity for the transportation of waste from harvesting site $i$ to pyrolysis facility $k$ with transportation mode $m$ at time period $t$

$Y_{k, g} \quad 0-1$ binary parameter, indicating whether intermediate type $g$ is processed in pyrolysis facility $k$

$\alpha_{b, p, q} \quad$ yield of product $p$ converted from unit quantity of poultry litter feedstock type $b$ at pyrolysis facilities with technology $q$

$\beta_{b, g, q} \quad$ yield of intermediate $g$ converted from unit quantity of poultry litter feedstock type $b$ at pyrolysis facilities with technology $q$

$\gamma_{g, p, q} \quad$ yield of product $p$ converted from unit quantity of intermediate type $g$ at intermediate upgrading facilities with technology $q$ '

$\varepsilon_{b, t} \quad$ proportion of poultry waste type $b$ deteriorated in storage facility at time $t$

$\eta_{l, q} \quad \quad$ minimum production amount as a proportion of capacity for intermediate upgrading facility $l$ with technology $q$ '

$\theta_{k, q} \quad$ minimum production amount as a proportion of capacity for pyrolysis facility $k$ with conversion technology $q$

$\rho_{b} \quad$ mass quantity of standard dry biomass of 1 dry ton of poultry waste type $b$

$\varphi_{p} \quad$ gasoline-equivalent gallons of 1 gal of product $p$

\section{Integer Variables}

$x_{k, q, r} \quad 0-1$ variable, equal to 1 if a pyrolysis facility with technology $q$ and capacity level $r$ is located at site $k$

$z_{l, q^{\prime}, r} \quad 0-1$ variable, equal to 1 if a upgrading facility with technology $q$ ' and capacity level $r$ is located at site $l$

\section{Non-Negative Continuous Variables}

$b m p_{b, i, t} \quad$ amount of poultry waste type $b$ procured from harvesting site $i$ in time period $t$ $\operatorname{capk}_{k, q, r}$ annual production capacity (in terms of dry tons of standard biomass) of pyrolysis 
facility $k$ with technology $q$ and capacity level $r$

capl $_{l, q^{\prime}, r} \quad$ annual production capacity (in terms of gallons of gasoline equivalent) of intermediate upgrading facility $l$ with technology $q$ ' and capacity level $r$

$f i k_{b, i, k, m, t} \quad$ amount of poultry waste type $b$ shipped from harvesting site $i$ to pyrolysis facility $k$ with transportation mode $m$ in time $t$

$f k d_{d, k, p, m, t}$ amount of product type $p$ shipped from pyrolysis facility $k$ to demand zone $d$ with transportation mode $m$ in time $t$

$f k l_{g, k, l, m, t} \quad$ amount of intermediate type $g$ shipped from pyrolysis facility $k$ to intermediate upgrading facility $l$ with transportation mode $m$ in time $t$

$f l d_{d, l, p, m, t}$ amount of product type $p$ shipped from intermediate upgrading facility $l$ to demand zone $d$ with transportation mode $m$ in time $t$

inck $k_{k} \quad$ incentive received for the construction of pyrolysis facility $k$

incl $l_{l} \quad$ incentive received for the construction of upgrading facility $l$

$r c v p k_{k} \quad$ annual processing cost of intermediate products in pyrolysis facility $k$

revpk $k_{k}$ annual processing emission of intermediate products in pyrolysis facility $k$

$r g k_{g, k, t} \quad$ intermediate product $g$ processed in pyrolysis facility $k$ in time $t$

$s b k_{b, k, t} \quad$ storage level of poultry waste type $b$ in pyrolysis facility $k$ at time $t$

$s g k_{g, k, t} \quad$ storage level of intermediate type $g$ in pyrolysis facility $k$ at time $t$

$s g l_{g, l, t} \quad$ storage level of intermediate type $g$ in intermediate upgrading facility $l$ at time $t$

$\operatorname{sold}_{d, p, t} \quad$ amount of product type $p$ sold to demand zone $d$ at time period $t$

$s p k_{k, p, t} \quad$ storage level of product type $p$ in pyrolysis facility $k$ at time $t$

$s p l_{l, p, t} \quad$ storage level of product type $p$ in upgrading facility $l$ at time $t$

tcapk $k_{k} \quad$ total capital investment of installing pyrolysis facility $k$

tcapl $_{l} \quad$ total capital investment of installing upgrading facility $l$

$t c f p k_{k} \quad$ fixed annual cost of pyrolysis facility $k$

$t c f p l_{l} \quad$ fixed annual cost of upgrading facility $l$

$w b k_{b, k, q, t} \quad$ amount of poultry waste type $b$ processed through pyrolysis technology $q$ in pyrolysis facility $k$ at time $t$

$w g k_{g, k, q, t} \quad$ amount of intermediate type $g$ produced through pyrolysis technology $q$ in pyrolysis facility $k$ at time $t$

$w g l_{g, l, q^{\prime}, t} \quad$ amount of intermediate type $g$ converted to liquid transportation fuel through 
conversion technology $q^{\prime}$ in intermediate upgrading facility $l$ at time $t$ $w p k_{k, p, q, t} \quad$ amount of product type $p$ produced through pyrolysis technology $q$ in pyrolysis facility $k$ at time $t$

$w p l_{l, p, q^{\prime}, t} \quad$ amount of product type $p$ produced through upgrading technology $q^{\prime}$ in upgrading facility $l$ at time $t$ 


\section{Parameter Values}

Table S1 Parameters in the life cycle optimization model.

\begin{tabular}{ccc}
\hline Parameters & Values & Unit \\
\hline$M C$ & 0.2281 & - \\
$I R$ & 0.1 & - \\
$N Y$ & 20 & year \\
$C B M_{b, i, t}$ & 7.76 & $\$ /$ ton \\
$C N O K$ & 7.041 & $\$ /$ ton syngas \\
$P R I_{d, \text { biochar }, t}$ & 100 & $\$ /$ ton \\
$P R I_{d, \text { diesel }, t}$ & 2.288 & $\$ / \mathrm{GEG}$ \\
$P R I_{d, \text { gasoline, }}$ & 2.265 & $\$ / \mathrm{GEG}$ \\
$I N C V_{d, \text { diesel }}$ & 0.26 & $\$ / \mathrm{GEG}$ \\
$I N C V_{d, \text { gasoline }}$ & 0.26 & $\$ / \mathrm{GEG}$ \\
\hline
\end{tabular}

Table S2 Reaction related parameters in the life cycle optimization model.

\begin{tabular}{ccc}
\hline Parameters & Values & Unit \\
\hline$\alpha_{b, \text { biochar,fast pyrolysis }}$ & 0.1907 & - \\
$\alpha_{b, \text { biochar, slow pyrolysis }}$ & 0.55 & - \\
$\beta_{b, \text { bio-oilfast pyrolysis }}$ & 0.1006 & kilogallon/ton \\
$\beta_{b, \text { bio-oil,slow pyrolysis }}$ & 0.0440 & kilogallon/ton \\
$\beta_{b, \text { syngas,fast pyrolysis }}$ & 0.136 & - \\
$\beta_{b, \text { syngas,slow pyrolysis }}$ & 0.25 & - \\
$\varepsilon_{b, t}$ & 0.037 & - \\
$\eta_{l, q}$ & 0.5 & - \\
$\theta_{k, q}$ & 0.5 & - \\
$\varphi_{\text {gas }}$ & 1 & - \\
$\varphi_{\text {diesel }}$ & 1.14 & - \\
\hline
\end{tabular}

Table S3 Computation related parameters in the life cycle optimization model.

\begin{tabular}{ccc}
\hline Parameters & Values & Unit \\
\hline$W C I K$ & 100 & million ton \\
$M D S$ & 200 & $\mathrm{~km}$ \\
$H Y$ & 360 & day \\
$H_{t}$ & 90 & day \\
$S K_{k, t}$ & 10 & day \\
$S L_{l, t}$ & 10 & day \\
$U P G K$ & 100,000 & million gallon \\
\hline
\end{tabular}


Table S4 Parameters in the life cycle optimization model.

\begin{tabular}{|c|c|c|}
\hline Parameters & Values & Unit \\
\hline$D F C B_{b, m}$ & 5 & \$/ton \\
\hline$D V C B_{b, m}$ & 0.18 & $\$ /($ ton•km) \\
\hline$D F C G_{g, m}$ & 5.67 & \$/kilogallon \\
\hline$D V C G_{g, m}$ & 0.119 & $\$ /($ kilogallon $\bullet \mathrm{km})$ \\
\hline$D F C P_{m, \text { gasoline }}$ & 3.28 & \$/kilogallon \\
\hline$D V C P_{m, \text { gasoline }}$ & 0.425 & $\$ /($ kilogallon $\bullet \mathrm{km})$ \\
\hline$D F C P_{m, \text { diesel }}$ & 3.28 & \$/kilogallon \\
\hline$D V C P_{m, \text { diesel }}$ & 0.425 & $\$ /($ kilogallon $\bullet k m)$ \\
\hline$D F C P_{m, \text { biochar }}$ & 5 & $\$ /$ ton \\
\hline$D V C P_{m \text { biochar }}$ & 0.18 & $\$ /($ ton $\bullet \mathrm{km})$ \\
\hline
\end{tabular}

Table S5 Parameter related to $\mathrm{CO}_{2}$ emissions in the life cycle optimization model.

\begin{tabular}{|c|c|c|}
\hline Parameters & Values & Unit \\
\hline $\mathrm{EBM}_{b, \mathrm{i}, \mathrm{t}}$ & 0.00596 & kton $\mathrm{CO}_{2} /$ kton \\
\hline $\mathrm{EHB}_{\mathrm{b}, \mathrm{t}}$ & 0.00051 & kton $\mathrm{CO}_{2} /$ kton \\
\hline $\mathrm{EHG}_{\mathrm{g}, \mathrm{t}}$ & 0.00000 & kton $\mathrm{CO}_{2} /$ million gallon \\
\hline $\mathrm{EHP}_{\text {biochar,t }}$ & 0.00000 & kton $\mathrm{CO}_{2} /$ kton \\
\hline $\mathrm{EHP}_{\text {diesel,t }}$ & 0.00000 & kton $\mathrm{CO}_{2} /$ million gallon \\
\hline $\mathrm{EHP}_{\text {gasoline,t }}$ & 0.00000 & kton $\mathrm{CO}_{2} /$ million gallon \\
\hline $\mathrm{ELD}_{\mathrm{d}, \mathrm{p}}$ & 0.00000 & kton $\mathrm{CO}_{2} /$ million gallon \\
\hline $\mathrm{ENOK}_{\mathrm{k}, \mathrm{g}}$ & 0.00000 & kton $\mathrm{CO}_{2}$ /kton \\
\hline $\mathrm{EPK}_{\mathrm{k}, \text { slow pyrolysis }}$ & 0.09720 & kton $\mathrm{CO}_{2}$ /kton \\
\hline $\mathrm{EPK}_{\mathrm{k}, \text { fast pyrolysis }}$ & 0.17075 & kton $\mathrm{CO}_{2} /$ kton \\
\hline $\mathrm{ETRB}_{\mathrm{b}, \mathrm{m}}$ & 0.00017 & kton $\mathrm{CO}_{2} /(\mathrm{kton} \bullet \mathrm{km})$ \\
\hline $\mathrm{ETRG}_{\mathrm{g}, \mathrm{m}}$ & 0.00032 & kton $\mathrm{CO}_{2} /($ million gallon $\bullet \mathrm{km})$ \\
\hline ETRP $_{\mathrm{m} \text {,diesel }}$ & 0.00004 & kton $\mathrm{CO}_{2} /($ million gallon $\bullet \mathrm{km})$ \\
\hline $\mathrm{ETRP}_{\mathrm{m} \text {,gasoline }}$ & 0.00004 & kton $\mathrm{CO}_{2} /($ million gallon $\bullet \mathrm{km})$ \\
\hline $\mathrm{ETRP}_{\mathrm{m}, \text { biochar }}$ & 0.00017 & kton $\mathrm{CO}_{2} /(\mathrm{kton} \bullet \mathrm{km})$ \\
\hline $\mathrm{ESCB}_{\mathrm{b}}$ & 0.00000 & kton $\mathrm{CO}_{2} /$ kton \\
\hline $\mathrm{ESCP}_{\text {biochar }}$ & 1.14253 & kton $\mathrm{CO}_{2} /$ kton \\
\hline
\end{tabular}




\section{Supplementary Figures}

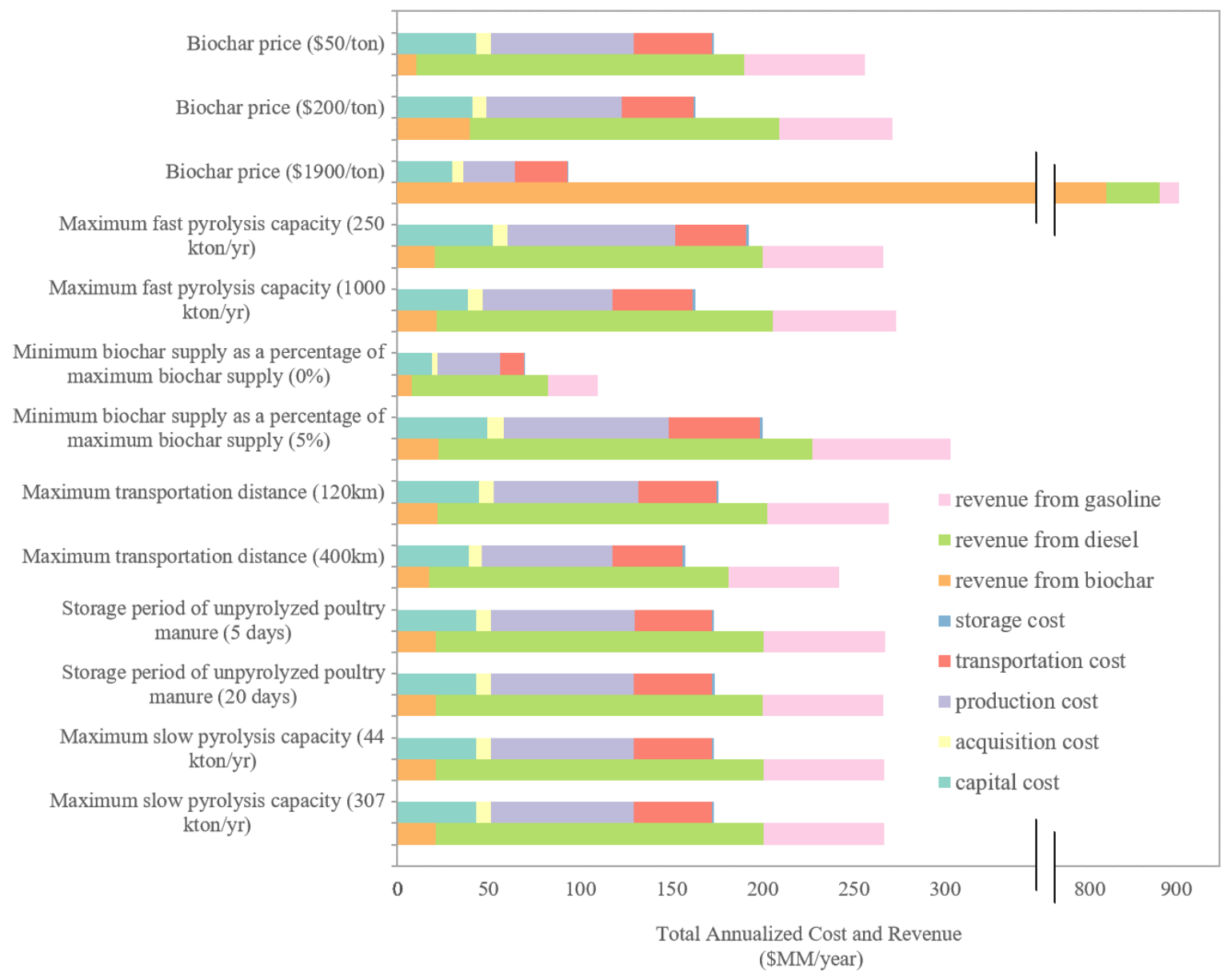

Figure S1. Economic breakdowns corresponding to the optimal supply chain networks on the changes of factors with the greatest effects on both objectives. 


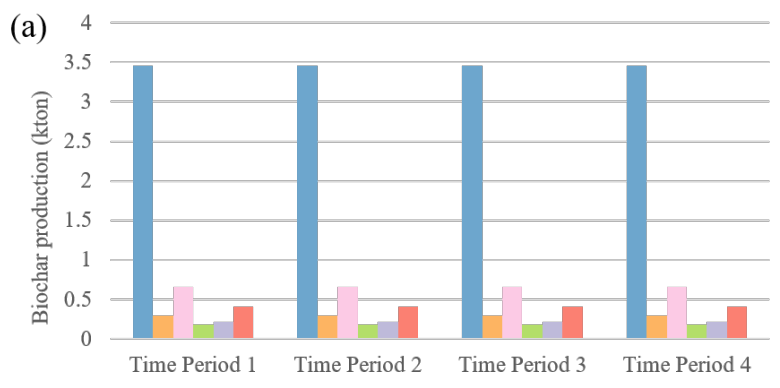

a Slow Pyrolysis Plant 1 - Slow Pyrolysis Plant 2 Slow Pyrolysis Plant 3

$\square$ Slow Pyrolysis Plant 4 - Slow Pyrolysis Plant 5 ø Slow Pyrolysis Plant 6

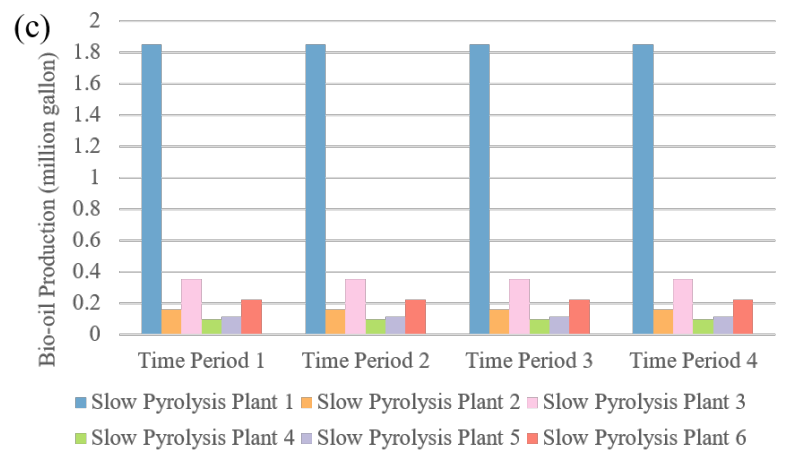

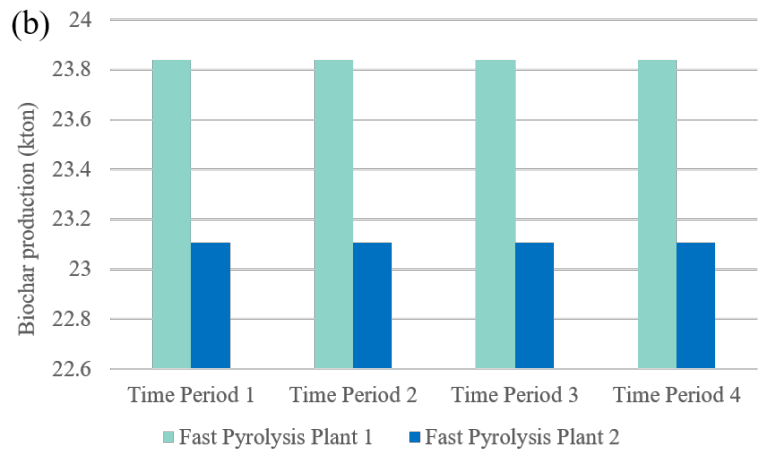

(d) 29.8

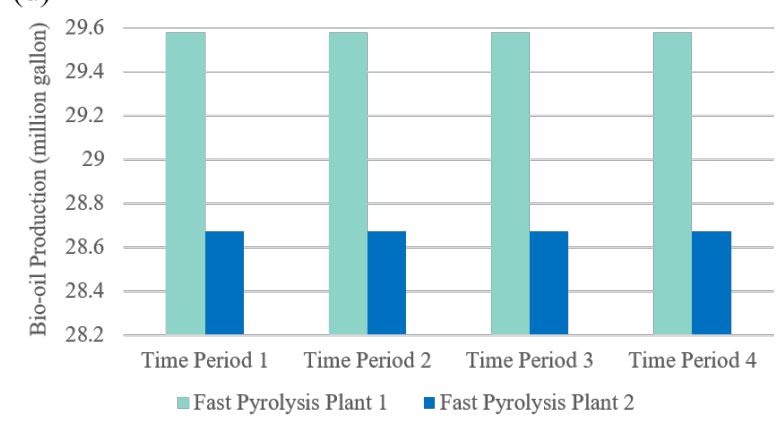

Figure. S2. Production planning of all pyrolysis plants for the economically optimal solution: (a) biochar production in each time period for slow pyrolysis plants; (b) biochar production in each time period for fast pyrolysis plants; (c) bio-oil production in each time period for slow pyrolysis plants; (d) bio-oil production in each time period for fast pyrolysis plants. 